\title{
Omega-3 Polyunsaturated Fatty Acids Role in Depression
}

ISSN: 2640-9208

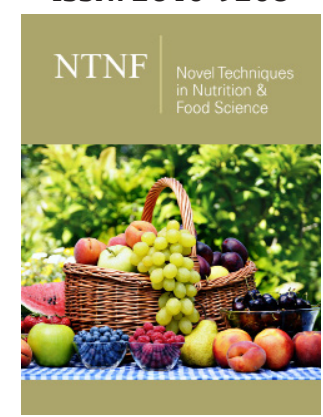

${ }^{* 1}$ Corresponding author: Edgar Díaz Acosta, Department of Biochemistry, México

Submission: 侮 May 13, 2019

Published: 眥 May 17, 2019

Volume 3 - Issue 5

How to cite this article: Acosta ED. Omega-3 Polyunsaturated Fatty Acids Role in Depression. Nov Tech Nutri Food Sci. 3(5).NTNF.000574.2019.

DOI: 10.31031/NTNF.2019.03.000574

Copyright@ Acosta ED, This article is distributed under the terms of the Creative Commons Attribution 4.0 International License, which permits unrestricted use and redistribution provided that the original author and source are credited.

\author{
Acosta ED* \\ Department of Biochemistry, México
}

\section{Introduction}

In recent years many investigations have been focused in the direct consumption of polyunsaturated fatty acids (PUFA) [1] in the prevention of inflammatory and cardiovascular diseases [2], polyunsaturated fatty acids Omega-3 (O3PUFA) represent a wide opportunity area in medical nutrition research, including eicosatetraenoic acid (EPA) emerging as a new potential substance in depression treatment [3]. Major depressive disorder (MDD) is a recurring disease characterized by sadness, loss of interest in activities and decreasing energy. Depression is a polygenic heterogeneous brain disorder, in which the mood of the person has been affected [1,4-7]. Polyunsaturated fatty acids (PUFA) are divided into two main groups or families: Omega-3 PUFA and Omega-6 PUFA [8], the main differences between these substances is the double bond position in the first carbon within the molecule. O3PUFA refers to a group in which the first double bond is 3 carbons from the end (omega) carbon atom of the molecule, while the 06PUFA are a family of PUFA that have in common a final carbon-carbon double bond in the n-6 position [9]. O3PUFA are synthetized by dietary shorter-chained omega-3 fatty acid alpha-linolenic acid (ALA) to form the more important long-chain omega-3 fatty acids: eicosatetraenoic acid (EPA) and docosahexaenoic acid (DHA) [1].

\section{Depression in the World and the USA}

According to the World Health Organization, it is estimated that 300 million people are affected by a depression state, near 800,000 persons die due to suicide every year. Suicide is the second cause of death among people 15-29 ages [10]. In the United States 17.3 million adults had at least one MDD in 2017 (this number represents $7.1 \%$ of all adults within the U.S.). The prevalence of MDD was higher in female (8.7\%), compared to male (5.3\%). On the other side, it is mentioned that the prevalence of MDD was higher in 2 or more races of adults (11.3\%), reported as shown in Figure 1 [11].

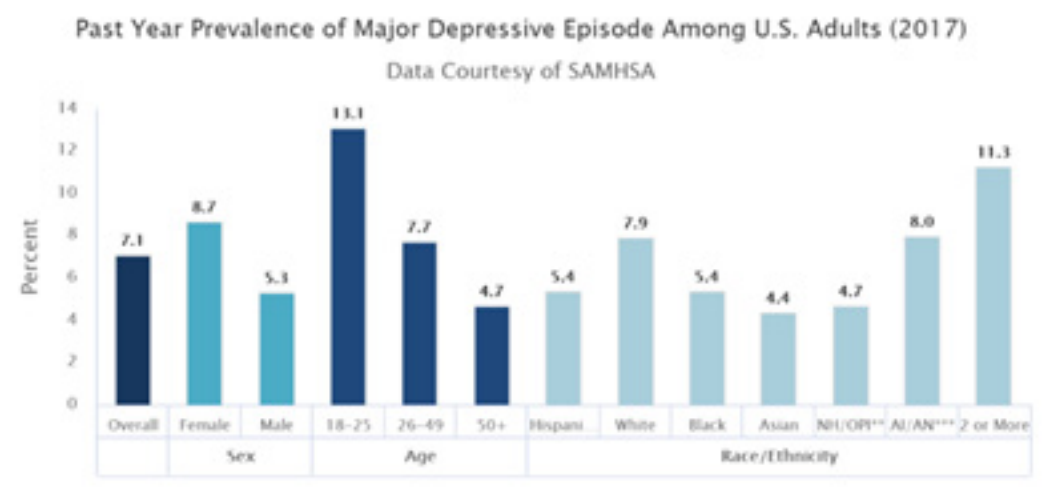

Figure 1: Credits: National institutes from mental health $\&$ substance abuse and mental health services administration 2019. 


\section{Treatment and Conclusion}

Patients with depression episodes are characterized by a low concentration of O3PUFA, it is well known that this fatty acid perform a key role in the central nervous system, as well as visual and cognitive functions. Carisha et al. [2] concluded in their study that patients with depressive episodes, the presence of 03PUFA low levels in blood circulation, compared to heathy patients, however there were no significative differences in the levels of 06PUFA [2]. The brain is considered a sensitive organ to oxidative damage, hereby the purpose to study the therapeutic use of 03PUFA as an antioxidant [8]. Several studies suggest that administration of 03PUFA $s$ has a favorable influence on major depressive disorders, Jahangarda et al. [10] showed that the depression symptoms decreased with O3PUFA administration in a study with $n=50$ patients, as well as anxiety and sleep symptoms $[12,13]$ in their study with Sixty five patients with co-morbidity of depression and overweight/obesity $(\mathrm{BMI} \geq 25)$ observed that omega-3 significantly reduced depression compared with the placebo $(\mathrm{P}=0.05)$ [14]. A great number of evidences in bibliography mentions the importance of EPA and DHA consumption in diet or as a dietary supplement, which could contribute to prevent and help in depression treatments [5,6,14-16]. Several strategies could help improve the neuroprotective activity of 03PUFA [8] and results show that the combination of 03PUFA and psychoeducation might be helpful to an improvement in symptoms in people with MDD or moderate depression [16].

\section{References}

1. Grosso G, Galvano F, Marventano S, Malaguarnera M, Bucolo C, et al (2014) Omega-3 fatty acids and depression: scientific evidence and biological mechanisms. Oxidative Medicine and Cellular Longevity, p. 16.

2. Thesing CS, Bot M, Milaneschi Y, Giltay EJ, Penninx BWJH (2018) Omega-3 and omega-6 fatty acid levels in depressive and anxiety disorders. Psychoneuroendocrinology 87: 53-62.

3. Logan A (2004) Omega-3 fatty acids and major depression: A primer for the mental health professional. Lipids Health Dis 3: 25.

4. Wani AL, Bhat SA, Anjum A (2015) Omega-3 fatty acids and the treatment of depression: a review of scientific evidence. Integr Med Res 4(3): 132141.
5. Logan A (2004) Omega-3 fatty acids and major depression: A primer for the mental health professional. Lipids Health Dis 3: 25.

6. Eckert GP, Lipka U, Muller WE (2013) Omega-3 fatty acids in neurodegenerative diseases: Focus on mitochondria. Prostaglandins Leukot Essent Fatty Acids 88(1): 105-114.

7. Kerdiles O, Layé S, Calon F (2017) Omega-3 polyunsaturated fatty acids and brain health: Preclinical evidence for the prevention of neurodegenerative diseases. Trends in Food Science \& Technology 69: 203-213.

8. Valenzuela R, Bascuñan K, Valenzuela A, Chamorro R (2009) Ácidos grasos omega 3 , enfermedades psiquiátricas y neurodegenerativas: un nuevo enfoque preventivo y terapéutico. Rev Chil Nutr 36(4): 11201128.

9. Jahangarda L, Sadeghia A, Ahmadpanaha M, Trachslerb EH, Bahmanib DS, et al. (2018) Influence of adjuvant omega-3-polyunsaturated fatty acids on depression, sleep and emotion regulation among outpatients with major depressive disorders - Results from a double-blind, randomized and placebo-controlled clinical trial. J Psychiatr Res 107: 48-56.

10. https://www.who.int/news-room/fact-sheets/detail/depression

11. https://www.nimh.nih.gov/health/statistics/major-depression.shtml

12. Keshavarz SA, Mostafavi SA, Akhondzadeh S, Mohammadi MR, Hosseini $S$, et al. (2018) Omega-3 supplementation effects on body weight and depression among dieter women with co-morbidity of depression and obesity compared with the placebo: A randomized clinical trial. Clin Nutr ESPEN 25: 37-43.

13. Sadeghi LJA, Ahmadpanah M, Trachsler EH, Bahmani DS, Haghighi M, et al. (2018) Influence of adjuvant omega-3-polyunsaturated fatty acids on depression, sleep and emotion regulation among outpatients with major depressive disorders - Results from a double-blind, randomized and placebo-controlled clinical trial. Journal of Psychiatric Research 107: 48-56.

14. Parletta N, Zarnowiecki D, Cho J, Wilson A, Procter N, et al. (2016) People with schizophrenia and depression have a low omega-3 index. Prostaglandins Leukot Essent Fatty Acids 110: 42-47.

15. Stahl LA, Begg DP, Weisinger RS, Sinclair AJ (2008) The role of omega-3 fatty acids in mood disorders. Curr Opin Investig Drugs 9(1): 57-64.

16. Tayama J, Ogawa S, Nakaya N, Sone T, Hamaguchi T, et al. (2019) Omega-3 polyunsaturated fatty acids and psychological intervention for workers with mild to moderate depression: A double-blind randomized controlled trial. J Affect Disord 245: 364-370. 